\title{
Should all patients receive statins before cardiac surgery: Are more data necessary?
}

Harold L. Lazar, MD

See related article on page 679.

From the Department of Cardiothoracic Surgery, the Boston Medical Center, and the Boston University School of Medicine, Boston, Mass.

Received for publication Sept 29, 2005; revisions received Oct 12, 2005; accepted for publication Oct 20, 2005.

Address for reprints: Harold L. Lazar, MD, Department of Cardiothoracic Surgery, Boston Medical Center, 88 East Newton St, Boston, MA 02118 (E-mail: harold.lazar@ bmc.org).

J Thorac Cardiovasc Surg 2006;131:520-2

$0022-5223 / \$ 32.00$

Copyright () 2006 by The American Association for Thoracic Surgery

doi:10.1016/j.jtcvs.2005.10.023
I $\mathrm{n}$ this issue of the Journal, Clark and coworkers ${ }^{1}$ conclude that pretreatment with statins before cardiac surgery decreases perioperative morbidity and mortality. The beneficial effects of statins were seen primarily in patients undergoing coronary artery bypass grafting (CABG). Although perioperative mortality was lower in valve patients receiving statins, this did not reach statistical significance, most likely because of reduced sample size.

There were several important limitations in the design of this study. It was retrospective and nonrandomized, and variable doses and types of statins were used. We are not told what the length of treatment was before surgical intervention. Furthermore, the dosages of statins were not targeted to achieve a specific lowdensity lipoprotein (LDL) level, as suggested by the most recent Adult Treatment Panel guidelines. ${ }^{2}$ In fact, LDL levels were not reported for either group, and therefore it is not known whether the beneficial effects of statins were due to cholesterol lowering or to their pleiotropic properties. Although propensity score analyses were used to match the patients, it is obvious that the patients who received statins also received a higher incidence of $\beta$-blockers, angiotensin-converting enzyme inhibitors, and aspirin therapy. In short, they had more complete cardioprotection, and although statistical analyses were undertaken to account for these discrepancies, the perception is that statin-treated patients were better prepared for surgical intervention and were more aggressively treated for risk factors by their internists and cardiologists. Studies have clearly demonstrated that patients receiving the combination of statins, angiotensin-converting enzyme inhibitors, $\beta$-blockers, and aspirin have significantly better survival after an acute coronary event.

Can we therefore conclude from this study that all patients undergoing cardiac surgery should receive statin therapy preoperatively? Should prospective randomized studies be performed to define the optimal dose, length of therapy, and specific statin for the cardiac surgical patient? Are further studies necessary to elucidate the mechanism for the beneficial effects of statins in the perioperative period? In short, are more data necessary?

\section{Favorable Effects of Statins in Patients With Coronary Disease}

Numerous clinical trials have shown that statin therapy improves survival and reduces ischemic events in patients with known coronary disease. These beneficial effects were seen irregardless of age, baseline LDL level, and the presence or absence of cardiac risk factors. In view of this, recent Adult Treatment Panel guidelines now recommend lowering LDL levels to less than $100 \mathrm{mg} / \mathrm{dL}$ in patients with known coronary disease and more aggressive statin therapy to lower LDL levels to less than $70 \mathrm{mg} / \mathrm{dL}$ for patients with coronary disease with the highest risk factors, which include diabetes, hypertension, obesity, smoking, the metabolic syndrome, and acute coronary syndromes (ACSs). ${ }^{2}$ The dose of the statin should be sufficient to achieve at least a $30 \%$ to $40 \%$ reduction in LDL levels. On the basis of these guidelines, all patients undergoing CABG should receive statin therapy before surgical intervention irregardless of their baseline LDL level. Experimental studies in a porcine CABG model have documented that the favorable effects of pretreatment with statins occur even in the face of relatively normal LDL values. 


\section{Statin Therapy in Patients Requiring Urgent and Emergency CABG}

Can statin therapy be equally effective in patients presenting with ACSs requiring urgent or emergency CABG in whom statin therapy can only be initiated less than 24 hours before surgical intervention? In a meta-analysis of 300,823 patients hospitalized for an acute myocardial infarction, the initiation of statin therapy within 24 hours of the infarction was associated with significantly lower rates of morbidity and mortality. ${ }^{3}$ Mortality was reduced from $15.4 \%$ to $4.0 \%$, and there was a lower incidence of cardiogenic shock, arrhythmias, cardiac arrest, and rupture. A recent review of 6 randomized controlled intervention trials involving patients with ACSs concluded that early initiation of high-intensity statin therapy $(80 \mathrm{mg} / \mathrm{d}$ atorvastatin) reduced recurrent ischemic events compared with moderate-intensity treatment (40 mg of pravastatin). ${ }^{4}$ Moderate-intensity regimens (40 $\mathrm{mg}$ of simvastatin, $20-40 \mathrm{mg}$ of pravastatin, $80 \mathrm{mg}$ of fluvastatin, and $0.4 \mathrm{mg}$ of cerivastatin) provided minimal benefit compared with placebo. The anti-inflammatory properties of intensive statin therapy might be responsible for these beneficial effects because high-dose $(80 \mathrm{mg})$ atorvastatin resulted in a $35 \%$ reduction of C-reactive protein (CRP) levels, with far less effects on LDL levels. These studies strongly suggest that all patients experiencing ACSs will benefit from intensive statin therapy, even if the drug is begun less than 24 hours from the time of infarction. Hence patients requiring urgent or emergency CABG will also benefit from the cardioprotection provided by statin therapy, even if initiated immediately before surgical intervention, provided that more intensive doses are used.

\section{Mechanisms for the Beneficial Effects of Statins in the Patient Undergoing CABG}

If statins exert their beneficial effects in the acutely ischemic patient without dramatic changes in LDL levels, what is the mechanism responsible for the cardioprotective effects of statins in patients with ACSs? Statins have been shown to work through intermediate pathways to improve endothelial function, reduce the inflammatory process, and decrease thrombosis independent of their LDL-lowering properties. Statins enhance endothelial function by decreasing superoxide formation, upregulating the expression of endothelial nitric oxide synthase, and inhibiting the release of angiotensin II and endothelin. They reduce inflammation by decreasing levels of CRP and reducing cytokine and adhesion molecule expression. Thrombosis is suppressed and fibrinolysis is enhanced by reducing platelet aggregation and adhesion and thromboxane formation and promoting fibrinolysis by enhancing tissue plasminogen activator synthesis and reducing tissue plasminogen activator inhibitor levels. These pleiotropic effects appear to be independent of LDL lowering and can occur within 24 hours of initiation of therapy but require higher doses of statins.

\section{Dosages, Types of Statins, and Safety Issues}

Most patients undergoing CABG surgery will require higher doses of statin therapy. However, are higher doses of statin therapy safe, and are all statins equal in their cardioprotective effects? Eighty milligrams of atorvastatin is the most common high-intensity statin used and has been associated with better outcomes in patients compared with moderate doses with other statins. Hence it is important to compare the safety of this dosage regimen with that of other statin agents.

The most serious side effect associated with statins is rhabdomyolysis. The highest risk for this complication was associated with cerivastatin (3.16 per million prescriptions), which is no longer on the market. The risk for other statins per million prescriptions is as follows: 0.19 for lovastatin, 0.12 for simvastatin and pravastatin, 0.04 for atorvastatin, and 0 for fluvastatin. The risk for rhabdomyolysis is associated with factors that increase serum concentrations of statins, such as small body size, advanced age, renal or hepatic dysfunction, diabetes, hypothyroidism, and drugs that interfere with statin metabolism, such as cyclosporin, antifungal agents, calcium-channel blockers, and amiodarone. High-dose atorvastatin has been associated with few side effects. In 44 atorvastatin trials, adverse events, such as liver dysfunction and myopathy, occurred with equal frequency in the placebo group and at each atorvastatin dose. In contrast, $80 \mathrm{mg}$ of simvastatin is associated with a low but definitive increased risk of myopathy $(0.4 \%)$. Hence the benefits of high-dose statin therapy appear to outweigh any potential risks.

\section{Statins and Valve Surgery}

Although the data are clear that statins benefit all patients undergoing $\mathrm{CABG}$, the data are less convincing for patients undergoing isolated valve surgery. There is evidence to suggest that the inflammatory response seen in atherosclerotic vascular disease might also be responsible for the progression of calcific deposits seen on native and bioprosthetic valves. Patients with high serum cholesterol levels have more rapid progression of aortic valve calcification and decreased aortic valve orifice areas. Aortic valve calcification has also been associated with higher levels of CRP and endothelial dysfunction. In the past, the cause of valve calcification has been thought to be degenerative processes, but there is now evidence to suggest that LDL receptor proteins might play an important role in osteoblast differentiation and skeletal bone formation. Atorvastatin significantly reduced aortic valve calcification in cholesterol-fed animals by reducing LDL receptor-related proteins responsible for osteoblast formation. Several clinical studies have suggested that statins might restrict the progression of aortic sclerosis, as assessed by means of echocardiography-derived aortic valve areas and mean gradients in dosages that had little 
effect on LDL lowering. Primary tissue failure of bioprosthetic valves is also related to calcification and inflammation similar to that seen in native valve disease. Farivar and $\mathrm{Cohn}^{5}$ found that patients with explanted bioprosthetic valves had higher cholesterol levels and a lower incidence of statin use. AntoniniCanterin and colleagues ${ }^{6}$ noted a decrease in annual mean gradients and the rate of decrease in effective orifice area in aortic bioprostheses in the presence of statin therapy. However, both of these studies were retrospective and nonrandomized, and the types of statins used, their dosages, and duration of therapy were not controlled. Specific levels of LDL were not targeted. Bioprosthetic valves show their greatest incidence of deterioration after 7 years; however, none of these studies exceeded a mean follow-up of 4 years. Therefore the true effect of statin therapy on the durability of bioprosthetic valves is unknown.

\section{Conclusions}

Should all patients receive statins before cardiac surgery, and are more data necessary? Data from numerous randomized clinical trials strongly suggest that all patients undergoing CABG should receive statins before surgical intervention. Patients undergoing elective operations should receive dosages of statins targeted to achieve LDL levels of less than $100 \mathrm{mg} / \mathrm{dL}$ and even lower $(<70 \mathrm{mg} / \mathrm{dL})$ for high-risk cases. Patients requiring urgent or emergency CABG should have high-dose statin therapy initiated as soon as the decision is made to proceed with the operation. Atorvastatin at $80 \mathrm{mg}$ would appear to be the statin of choice for these patients on the basis of its superior results in trials involving patients with ACSs. Statin therapy should be initiated in these patients, irregardless of their baseline LDL levels. On the basis of previous CABG trials showing significantly improved graft patency, decreased need for repeat revascularization, and lower incidence of ischemic cardiovascular events, all patients undergoing CABG should receive lifetime statin therapy. The benefits far outweigh the minimal risks of toxicity with these drugs.
The role of statin therapy remains less clear in patients undergoing isolated valve surgery. I suspect that statins will have a cardioprotective effect in patients undergoing isolated aortic and mitral valve replacement for calcific stenosis and will enhance the longevity of bioprostheses implanted in these patients. However, only prospective, multicenter, randomized trials with specified dosages of statins to achieve a target LDL level can provide the definitive answer for the role of statin therapy in these patients. These studies take time, are expensive, and might not be funded by pharmaceutical companies when patents are near expiration. In the meantime, I have begun to initiate statin therapy in patients with aortic and mitral stenosis and in all patients receiving a bioprosthetic valve, irregardless of their baseline LDL level. Although more data are necessary, the increased benefits of early cardioprotection and potential for enhanced bioprosthetic durability appear to outweigh the minimal risks involved with these drugs.

\section{References}

1. Clark LL, Ikonomidis JS, Crawford FA Jr, Crumbley A III, Kratz JM, Stroud MR. Preoperative statin treatment is associated with reduced postoperative mortality and morbidity in patients undergoing cardiac surgery: an 8-year retrospective cohort study. J Thorac Cardiovasc Surg. 2006;131:679-85.

2. Grundy SM, Cleeman JJ, Merz CNB, Brewer BJR, Clark LT, Hunninghake DB, et al. Implications of recent clinical trials for the National Cholesterol Education Program Adult Treatment Panel III Guidelines. Circulation. 2004;110:227-39.

3. Fonarow GC, Wright RS, Spencer FA, Frederick PD, Doug W, Every $\mathrm{N}$, et al. Effect of statin use within the first 24 hours of admission for acute myocardial infarction on early morbidity and mortality. Am J Cardiol. 2005;96:611-6.

4. Schwartz GC, Olsson AG. The case for intensive statin therapy after acute coronary syndromes. Am J Cardiol. 2005;96:45-53F.

5. Farivar RS, Cohn SH. Hypercholesterolemia is a risk factor for bioprosthetic valve calcification and explanation. $J$ Thorac Cardiovasc Surg. 2003;126:969-75.

6. Antonini-Canterin F, Zuppiroli A, Popescu BA. Effect of statins on the progression of bioprosthetic aortic valve degeneration. Am J Cardiol 2003;92:1479-82. 